\section{Céleste Lebbé}

\section{ADRESSE}

C. Lebbé : boursière post-doctorale de l'ANRS, dermatologue. Service de dermatologie du $\operatorname{Pr}$ Morel, hôpital Saint-Louis, 1, avenue Claude-Vellefaux, 75010 Paris, France et Laboratoire de pharmacologie du Pr Calvo, Institut de Génétique moléculaire, 27, rue Juliette-Dodu, 75010 Paris, France.

\title{
Caractéristiques cellulaires et moléculaires du sarcome de Kaposi
}

Le sarcome de Kaposi est caractérisé par la prolifération de cellules fusiformes dont l'origine, cellules endothéliales, dendritiques ou musculaires lisses, demeure controversée. La nature bénigne ou maligne de cette prolifération est également incertaine; elle pourrait être induite par différentes cytokines et facteurs de croissance conduisant parfois à une transformation tardive. Bien que de nombreux arguments épidémiologiques plaident en faveur de l'origine infectieuse de cette tumeur, les données microbiologiques étaient peu convaincantes jusqu'à la mise en évidence très récente dans les lésions kaposiennes d'un nouveau virus herpès, HHV-8. La présence de séquences d'HHV-8 dans le sang de patients infectés par le VIH est prédictive de la survenue ultérieure d'un sarcome de Kaposi, ce qui est un argument majeur en faveur du rôle causal de HHV-8.

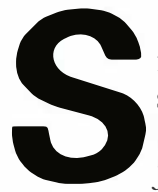

urvenant auparavant de façon endémique chez des sujets âgés, originaires du bassin méditerranéen, ou juifs d'Europe de l'Est, le sarcome de Kaposi (SK) est devenu épidémique dans les années 1980 chez certains patients infectés par le virus de l'immunodéficience acquise humaine (VH). La physiopathologie de la maladie reste mystérieuse même si d'intéressants modèles expérimentaux ont été mis au point [1]. La responsabilité d'un nouveau virus du groupe des $\gamma$-herpesviridae, a été récemment suggérée, offrant de nouvelles voies de recherche $\left(\mathrm{m} / \mathrm{s} n^{\circ} 6\right.$, vol. 11, p. 914; $n^{\circ} 10$, vol. 11, p. 1605) [2].

\section{Aspects clinicopathologiques}

Les formes clinico-épidémiologiques du sarcome de Kaposi [3, 4]

Le SK «classique" est rare, touchant des sujets entre 50 et 80 ans, et environ dix ou quinze hommes pour une femme, d'origine méditerranéenne ou juive d'Europe centrale. Quoique controversée, une association avec le phénotype HLA DR5 a été rapportée [5]. L'affection, caractérisée par des lésions cutanées prédominant aux extrémités, est d'évolution lente et l'atteinte viscérale, découverte fréquente d'autopsie, est rarement 


\section{RÉFÉRENCES}

1. Roth K, Brandstetter H, Stürzl M. Cellular and molecular features of HIV associated Kaposi's sarcoma. AIDS 1992 ; 6 : 895-913.

2. Chang Y, Cesarman E, Pessin M, Lee F, Culpepper J, Knowles D, Moore P. Identification on a new human herpes virus-like DNA sequences in AIDS-associated Kaposi's sarcoma. Science 1994; 266: 1865-9.

3. Janier M. Maladie de Kaposi au cours du SIDA. Rev Eur Dermatol MST 1991 ; 3: 59-77.

4. Friedman-Kien A, Saltzman B. Clinical manifestation of classical, endemic african and epidemic AIDS-associated Kaposi's sarcoma. I Am Acad Dermatol 1990; 22: 1238 50 .

5. Strichman Almashanu L, Weltfriend $\mathrm{S}$, Gideoni O, Friedman-Birnbaum R, Pollack $\mathrm{S}$. No significant association between HLA antigens and classic Kaposi's sarcoma: molecular analysis of 49 Jewish patients. J Clin Immunol 1995; 15: 205-9.

6. Farge D, Frances C. Quelle attitude à adopter devant un sarcome de Kaposi chez le transplanté d'organe? Néphrologie 1991; $12: 245-9$

7. Rabkin C, Yellin F. Cancer incidence in a population with a high prevalence of infection with immunodeficiency virus type $I$. J Natl Cancer Inst 1994; 86: 1711-6.

8. Ioannidis J, Skolnik P, Chalmers T, Lan J. HLA associations of epidemic Kaposi's sarcoma. AIDS 1995; 9: 649-64.

9. Friedman-Birnbaum R, Weltfriend S, Pollack S. Classic Kaposi's sarcoma: T-lymphocyte subsets, T4/T8 ratio, and NK cell activity. J Am Acad Dermatol 1991 ; 24 : 937-40.

10. Marinig C, Fiorini G, Boneschi V, Melotti E, Brambilia L. Immunologic and immunogenetic features of primary Kaposi's sarcoma. Cancer 1985 ; 55: 1899 901.

11. Kestens L, Melbye M, Biggar R. Endemic african Kaposi's sarcoma is not associated with immunodeficiency. Int $J$ Cancer $1985 ; 36$ : 49-54.

12. Chor PJ, Santa Cruz D. Kaposi's sarcoma. I Cutan Pathol 1992; 19: 6-20.

13. Kanitakis J, Roca-Miralles M. FactorXIIIa-expressing dermal dendrocytes in Kaposi's sarcoma. Virchows Archiv A Pathol symptomatique. On peut mentionner la description récente de SK chez. des patients homosexuels non infectés par le VIH et sans altérations, en règle, de leur bilan immunitaire [4]. Le SK africain, d'aspect parfois comparable au SK classique, réalise plus souvent des lésions infiltrantes aggressives localement ou disséminées avec atteintes cutanéomuqueuse et viscérale. Les formes dites lymphadénopathiques sans atteinte cutanée, fatales en un à trois ans, sont particulières à l'enfant en période prépubertaire [3].

Les SK les plus fréquents touchent aujourd'hui les sujets immunodéprimés. Des SK sont décrits en cas d'immunosuppression iatrogène. Cette tumeur survient en moyenne 15 à 22 mois après une transplantation d'organe, chez $0,45 \%$ des transplantés hépatiques, $0,41 \%$ des transplantés cardiaques et $1,24 \%$ des transplantés hépatiques dans une étude menée en Île-de-France. Ces SK sont régressifs à l'arrêt ou à la levée de l'immunosuppression dans environ $50 \%$ des cas dans les formes cutanées mais seulement dans $18 \%$ des cas dans les formes viscérales [6]. Le SK du SIDA, ou SK épidémique survient préférentiellement chez des hommes jeunes (dans la proportion de huit hommes pour une femme) et, dans $95 \%$ des cas, chez des homosexuels. L'incidence (25/100 000/an dans une population d'hommes mariés et 540/100 000/an dans une population d'homosexuels) est stable depuis 10 ans mais le diagnostic est actuellement porté surtout à des phases tardives de l'infection par le VIH [7]. Une méta-analyse récente conclut à l'absence d'association avec le phénotype HI_A DR3 et une possible association avec les phénotypes HLA B35 et Cw4 [8].

Le SK du SIDA est plus agressif que le SK classique avec une atteinte cutanée plus ubiquitaire, une atteinte muqueuse et viscérale plus fréquente. La survie est corrélée au taux de lymphocytes CD4 circulants et le rapport CD4/CD8, la présence de signes généraux et d'infections opportunistes [3]. Plutôt qu'un véritable cancer, le SK est actuellement considéré comme une «néoplasie opportuniste». L'exérèse précoce d'une lésion n'empêche pas l'apparition d'autres localisations. Le pro- nostic est corrélé à l'état immunitaire du patient et non au nombre de lésions [3].

\section{Caractéristiques immunologiques des malades}

Outre les modifications du taux de lymphocytes CD4 et du rapport CD4/CD8, d'autres anomalies immunologiques ont été rapportées au cours du SK épidémique: une diminution des tests de prolif ération lymphocytaire en présence de mitogènes et d'alloantigènes; une diminution de l'activité natural killer (NK) circulante; un défaut de production d'IL2 et d'interféron par les cellules mononucléées sanguines stimulées par la phytohémagglutinine $\mathrm{A}$, peut être lié à une anomalie fonctionnelle des monocytes [3].

Au cours du SK classique ni le taux des lymphocytes $\left(\mathrm{D} 4^{+}\right.$, ni le rapport (D4/CD8 ne sont modifiés, en règle générale, en dehors d'une discrète augmentation des lymphocytes $\mathrm{CD}^{+}$ et d'une diminution du rapport CD4/CD8 dans quelques formes cutanées diffuses ou systémiques [9]. Des résultats divergents ont été obtenus par Marinig el al. [10] qui rapportent une diminution du taux des lymphocytes $\mathrm{CD}^{+}$circulants et une augmentation du rapport (:D4/CD8, le taux de lymphocytes $\mathrm{CD}^{+}{ }^{+}$restant normal. Enfin une diminution de l'activité natural killer (NK) a été signalée, non corrélée à la gravité de la maladie [9]. Une étude portant sur 41 cas de SK africain a montré une augmentation du nombre de lymphocytes $\mathrm{CD}^{+}$circulants sans modification significative du rapport CD4/CD8 [11].

\section{Caractéristiques histologiques et immunohistochimiques}

Quel que soit le stade de la maladie, la cellule fusiforme représente la cellule kaposienne. Les caractéristiques histologiques semblent identiques, qu'il s'agisse du SK classique et du SK associé au VIH [12]. Dans la forme avancée, au stade nodulaire [12], on observe un mélange de structures vasculaires et de cellules fusiformes formant des nappes qui ménagent des fentes vasculaires; ces fentes n'ont généralement pas d'endothélium bien caractérisé; les 
cellules fusiformes ont une activité mitotique variable et présentent parfois quelques atypies cytologiques modérées. Un infiltrat inflammatoire mononucléé, souvent riche en plasmocytes est présent; enfin, on note en périphérie des nodules une prolifération de vaisseaux capillaires et lymphatiques (figure 1A). Au stade débutant, maculeux, on distingue de petits foyers nodulaires contenant quelques cellules fusiformes, des néovaisseaux, mais surtout un infiltrat inflammatoire avec prédominance de lymphocytes et présence de plasmocytes périvasculaires (figure 1B) [12]. Cet infiltrat diminue au cours de l'évolution du SK classique; il serait significativement moindre au cours du SK survenant chez des patients immunodéprimés avec, en particulier, un effondrement du nombre des CD4 au sein de l'infiltrat [13]. En microscopie électronique, les vaisseaux perdent certaines caractéristiques d'un endothélium veineux : fragmentation puis disparition de la membrane basale, disparition des péricytes, diminution des jonctions intercellulaires qui deviennent rudimentaires, diminution puis disparition du nombre de corps de Weibel-Palade [1].

L'origine de la cellule kaposienne n'est toujours pas élucidée: s'agit-il de myofibroblastes, de péricytes ou de cellules musculaires lisses, de cellules dendritiques, de ccllules endothéliales? L'hypothèse de l'origine endothéliale est étayée par l'expression en histochimie ou immunohistochimie d'antigènes et de lectines endothéliaux Ulex Europeus I Agglutinin (dont l'expression est inconstante), EN-4, BMA 120, CD34, thrombomoduline, ELAM1 (endothelial leucocyte adhesion molecule 1), collagène IV, laminine; l'expression du facteur Willebrand est inconstamment retrouvée [1, 14]. Pour d'autres, les cellules kaposiennes dériveraient de cellules dendritiques en raison de l'expression du facteur XIII, de la molécule d'adhérence I.FA1, et parfois du CD4 [15], mais ces données restent controversées [13, 14]. Enfin, pour Weich et al. [16] la présence d' $\alpha$ actine de muscle lisse dans certaines cellules fusiformes constituerait un argument en faveur de leur origine myofibroblastique ou péricytaire, mais ces
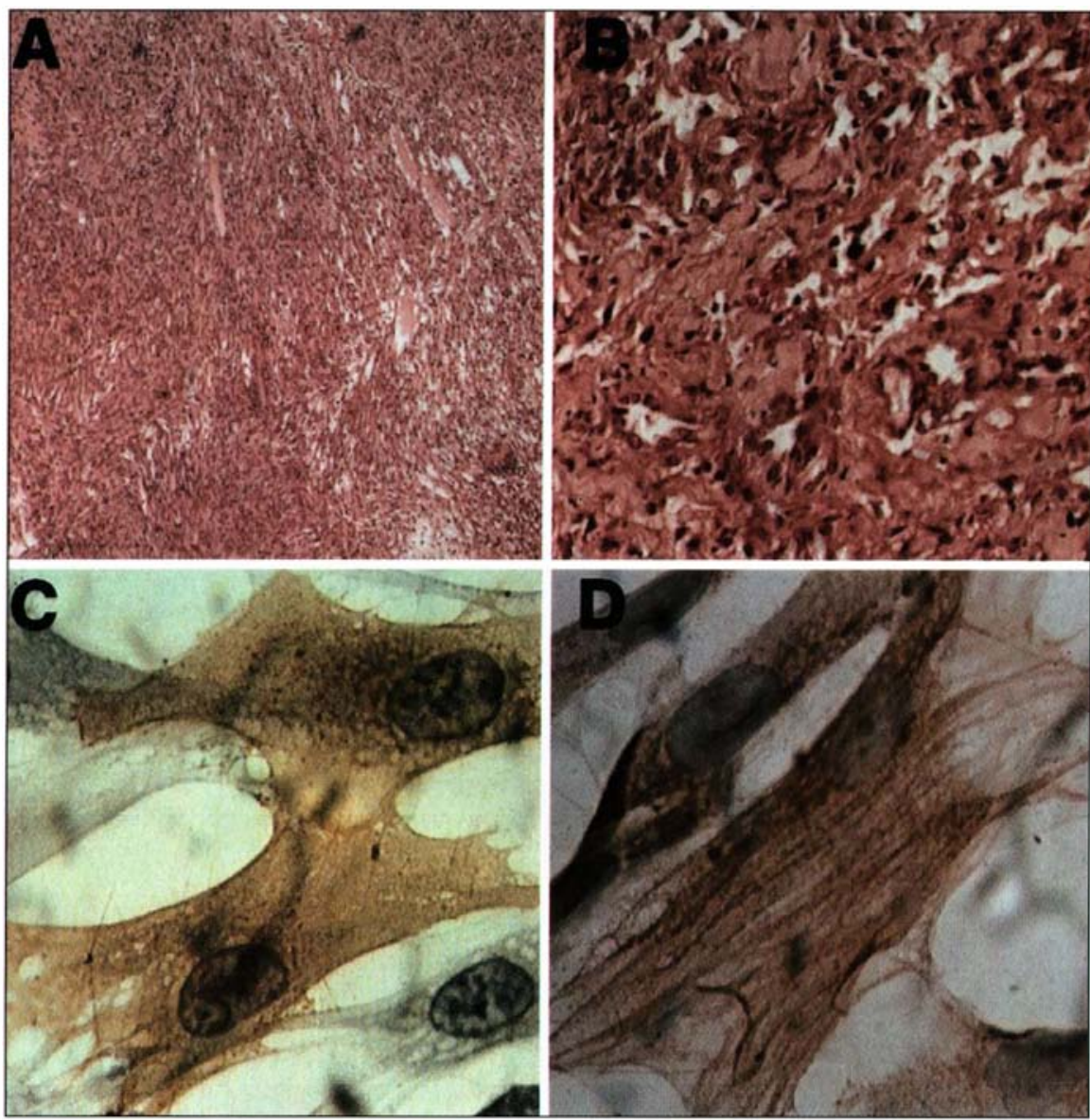

Figure 1. Caractéristiques histologiques et immunohistochimiques des sarcomes de Kaposi (SK). A. Coupe histologique de SK dans une forme nodulaire (HE x 4). Cellules fusiformes formant des nappes qui ménagent des fentes vasculaires. B. Coupe histologique de SK dans une forme débutante $(H E \times 20)$ : on observe quelques foyers de cellules fusiformes, des néovaisseaux mais surtout un infiltrat inflammatoire lymphoplasmocytaire. C. Marquage de cellules fusiformes kaposiennes en culture avec l'anticorps antiCD36 (x 1000). D. Marquage de cellules fusiformes kaposiennes en culture avec l'anticorps anti- $\alpha$-actine de muscle lisse (x 1000).

résultats sont contestés [14]. L'ensemble de ces données souligne l'hétérogénité phénotypique au sein d'une même tumeur kaposienne et aucune conclusion définitive ne peut actuellement être apportée quant à l'origine des cellules fusiformes.

\section{Oncogènes et virus impliqués dans le sarcome de Kaposi}

L'analyse caryotypique des tumeurs est, en règle, normale [17] bien qu'une étude récente suggère l'existence plus fréquente d'aneuploïdie dans les SK induits par les immunosuppresseurs [18]. La recherche de modifications de différents oncogènes ou anti-oncogènes [1] est, jusqu'à présent, toujours restée négative sur des tumeurs kaposiennes, ainsi que sur les modèles cellulaires établis à partir de ces tumeurs à l'exception de quatre études: Delli Bovi et al. [19] ont isolé, après transfection d'ADN de SK à des cellules 3T3, un nouvel oncogène appartenant à la famille des FGF (fibroblast growth factor), mais un artefact lié à la manipulation in vitro de l'ADN n'est 


\section{RÉFÉRENCES}

14. Regezi J, Mac Phail L, Daniels T, DeSouza Y, Greenspan J, Greenspan D. HIV-associated oral Kaposi's sarcoma. A heterogeneous cell population dominated by spindle-shaped endothelial cells. $\mathrm{Am}$ JPathol 1993; 143: 240-9.

15. Nickoloff B, Griffith C. The spindle-shaped cells in cutaneous Kaposi's sarcoma. Am J Pathol 1989; 135 : 793-800.

16. Weich $H$, Salahuddin S, Gill P, Nakamura S, Gallo R, Folkman J. AIDS associated Kaposi's sarcoma derived cells in long term cultures express and synthesize smooth muscle alpha-actin. Am J Pathol $1991 ; 139: 1251-8$.

17. Bisceglia M, Bosman C, Quirke P. A histologic and flow cytometric study of Kaposi's sarcoma. Cancer 1992; 69: 793-8.

18. Reizis Z, Trattner A, Katzenelson V, David M, Rotem A, Nativo M, Mor Y. Flow cytometric DNA analysis of classic and steroid induced Kaposi's sarcoma. Br J Dermatol 1995; 132 : 548-50.

19. Delli Bovi P, Curatola A, Kern F, Greco A, Ittmen M, Basilico C. An oncogene isolated by transfection of Kaposi's sarcoma DNA encodes a growth factor that is a member of the FGF family. Cell 1987; 50: $729-37$.

20. Huang Y, Li J, Moscatelli D, Basilico C, Nicolaides A, Zhang W, Poiesz B, FriedmanKien A. Expression of int-2 oncogene in Kaposi's sarcoma lesions. J Clin Invest 1993; 91:1191-7.

21. Nicolaides A, Huang $\mathrm{Y}, \mathrm{Li} \mathrm{J}$, Zhang $\mathrm{W}$, Friedman-Kien A. Gene amplification and multiple mutations of the K-ras oncogene in Kaposi's sarcoma. Anticancer Res 1994; 14: $921-6$.

22. Scinicariello F, Dolan M, Nedelcu I, Tyring S, Hilliard J. Occurrence of human papillomavirus and p53 gene mutations in Kaposi's sarcoma. Virology $1994 ;$; 203 : 153-7.

23. Rabkin C, Bedi G, Musaba E, Sunkuiu R, Mwansa N, sidransky D, Biggar R. AIDSrelated Kaposi's sarcoma is a clonal neoplasm. Clin Cancer Res 1995; 1 : 257-60.

24. Jacobson L, Armenian H. An integrated approach to the epidemiology of Kaposi's

cependant pas exclu; Huang et al. [20] ont montré l'expression de l'oncogène int-2 dans $55 \%$ des lésions de malades atteints de SK épidémique et retrouvé une mutation ponctuelle dans 8 des 9 cas étudiés; une mutation du gène $K$-ras a été observée dans sept cas sur trente et un de SK, associés ou non au VIH, et une amplification de ce gène dans $10 \%$ des cas étudiés [21]; enfin, une mutation hétérozygote du gène codant pour p53 n'a été montrée que par une seule équipe [22]. Plus récemment une étude de clonalité du SK a été effectuée, fondée sur l'inactivation aléatoire du chromosome X chez la femme, en utilisant le polymorphisme du gène du récepteur des androgènes [23]. Un profil d'inactivation monoclonal a pu être montré dans deux des trois cas qui ont pu être évalués; néanmoins, nous n'avons pas confirmé ces données malgré l'utilisation de techniques semblables chez sept femmes souffrant de SK (Delabesse E, Oksenhendler E, Lebbé C, Verola $\mathrm{O}$, Varet B, Turhan A, comm̂nucation personnelle).

Les arguments les plus convaincants en faveur de l'origine infectieuse du SK sont d'ordre épidémiologique: tout d'abord, la fréquence accrue chez les patients immunodéprimés et la survenue possible de SK chez des patients homosexuels non infectés par le virus VIH [24]; au cours du SIDA, le risque de développer un SK est plus élevé chez les homosexuels que dans les autres populations à risque pour le VIH et est, d'ailleurs, corrélé au nombre de partenaires sexuels masculins et aux antécédents de maladies sexuellement transmissibles du malade; il est aussi associé aux rapports oroanaux; enfin, parmi les femmes infectées par le virus $\mathrm{VIH}$, le risque de développer un SK est quatre fois plus élevé chez celles qui ont des rapports sexuels avec des sujets bisexuels qu'en cas de partenaire hétérosexuel.

L'observation, en microscopie électronique, de particules virales, de type herpesvirus, ou rétrovirales avait été parfois rapportée [1]; néanmoins, les données microbiologiques étaient restées, jusqu'en décembre 1994, peu concluantes: le cytomégalovirus (CMV) a été suspecté bien avant l'épidémie du SIDA sur des arguments sérologiques, sur la mise en évidence (inconstante) de virions ou de virus mûrs au sein de lésions de SK ou sur la présence d'ADN viral et d'antigènes structuraux en l'absence de virions; cependant d'autres tentatives d'hybridation avec des sondes ADNc spécifiques du CMV se sont avérées négatives ou inconstamment positives et n'intéressant pas les cellules fusiformes [1]. Le rôle pathogène direct du virus VIH n'a jamais été démontré. La production de lésions évocatrices de SK chez des souris transgéniques pour le gène tat du VIH est intéressante mais n'a jamais été confirmée [25]. Le gène tat n'est pas exprimé par des sarcomes de Kaposi de patients non infectés par le VIH [26]. Quelques cas de SK ont été rapportés chez des patien ts porteurs, dans leurs lymphocytes périphériques, de séquences du virus HTLVI, soit de façon isolée chez deux patients homosexuels, soit en association à un lymphome [27]. Cependant, la recherche de séquences HTLVI dans les lymphocytes périphériques est restée négative chez 17 patients sur 19 souffrant de SK non associé au VIH [27] et nous pensons que ce virus n'est pas associé de façon significative au SK.

D'autres agents infectieux ont également été mis en cause mais inconstamment retrouvés: le rôle de certains papillomavirus (HPV 16 et 18) a été suspecté sur des données de PCR effectuées à partir de tumeurs et de cellules en culture [28]. Néanmoins le caractère très inconstant des données $(20 \%$ des prélèvements positifs) et la nécessité de recourir à la PCR conduit aux mêmes réserves que celles énoncées pour le CMV. Ces résultats ont d'ailleurs par la suite été remis en question [29]. Des séquences d'HHV6 (human herpes virus 6) de type $\mathrm{A}$ ou de type $\mathrm{B}$ ont été détectées par PCR dans $30 \%$ des tumeurs kaposiennes analysées mais la positivité plus fréquente en peau saine que dans les tumeurs semble plutôt le reflet d'une infection virale disséminée [30]. Enfin une association à la présence d'anticorps sériques dirigés contre Mycoplasma penetrans chez des patients homosexuels souffrant de SK associé au SIDA a été suggérée puis infirmée [31]. Les recherches virales sur des modèles in vitro (développés plus 


\section{RÉFÉRENCES}

25. Vogel J, Hinrichs S, Reynolds R, Luciw $P$, Jay $G$. The HIV tat gene induces dermal lesions resembling Kaposi's sarcoma in transgenic mice. Natue 1988; 335:601-11.

26. Huang Y, Buchbinder A, Li J, Nicolaides A, Zhang C, Friedman-Kien A. The absence of Tat sequences in tissues of HIV-negative patients with epidemic Kaposi's sarcoma. AIIDS $1992 ; 6$ : 1139-42.

27. Lebbé C, Agbalika F, De Crémoux P, Deplanche $\mathbf{M}$, Masgrau E, Rybojad M, Morel P, Calvo F. Detection of KSHV and HTLV I sequences in HIV unrelated Kaposi's sarcoma. Arch Dermatol 1996 (sous presse).

28. Huang Y, Li J, Rush M, Poiesz BJ, Nicolaidis $A$, Jakobson $M$, Zhang $W G$, Coutavas E, Abbott MA, Friedman-Kien AE. HPV-16related DNA sequences in Kaposi's sarcoma. Lancet 1992; 339:515-8.

29. Kaaya E, Voevodin A, Szaecki P, Biberfeld P. No evidence of HPV in epidemic and endemic KS. I Acquir Immune Defic Syndr Hum Retrovirol 1993; 6 : 964-7.

30. Kempf W, Adams V, Pfaltz M, Briner J, Schmid M, Moos R, Hassam S. Human herpes virus type 6 and cytomegalovirus in AIDS-associated Kaposi's sarcoma. Hum Pahol 1995; 26 : 914-9.

31. Grau O, Slizewicz B, Tuppin P, Launay $V$, Bourgeois E, Sagot N, Moynier M, Lafeuillade A, Bachelez H, Clauvel JP, Blanchard A, Bahraoui E, Montagnier L.. Association of Mycoplasma penetrans with HIV infection. J Infect Dis $1995 ; 172: 672-81$.

32. Siegal B, Levinton-Kriss S, Schiffer A, Sayar J, Engelberg I, Vonsoyer A, Ramon Y, Rubinstein E. Kaposi's sarcoma in immunosuppression possibly the result of a dual viral infection. Cancer 1990; 65: 492-8.

33. Dupin N, Grandadam M, Calvez V, Gorin I, Aubin I, Havard S, Lamy S, Leibowitch $M$, Hureaux J, Escande J, Agut $H$. Herpesvirus-like DNA sequences in patients with Mediterranean Kaposi's sarcoma. Lancet $1995 ; 345: 761-2$

34. Soulier J, Grollet L, Oksenhendler E, Cacoub P, Carals-Hatem D, Babinet P, d'Agay MF, Clauvel JP, Raphael M, Degos L, Signaux F, Kaposi's sarcoma-associated herpesvirus-like DNA sequences in multicentric Castleman's disease. Blood 1995; 86: 1276 80.

35. Whitby D, Howard M, Tenant-Flowers M, Brink N, Copas A, Boshoff C, Hatzioannou $T$, Suggett F, Aldam D, Denton A, Miller R, Weller I, Weiss R, Tedder R, Schulz $T$. Detection of Kaposi's sarcoma associated herpesvirus in peripheral blood of HIVinfected ndividuals and progression to Kaposi's sarcoma. Lancet 1995; 346: 799802 .

loin) sont restées négatives hormis trois exceptions: (1) mise en évidence de germes apparentés à des mycoplasmes dans les cellules 3T3 transformées après transfection d'ADN extrait de SK (un artefact de culture n'est cependant pas exclu) [1]; (2) présence de particules virales (CMV et un autre virus non identifié en microscopie électronique) dans des surnageants de cellules kaposiennes obtenues à partir de SK d'un patient transplanté rénal [32] ; (3) enfin détection par PCR de séquences d'HPV 16 [28].

A l'inverse de cette recherche fondée sur l'identification de micro-organismes déjà connus dans des lésions kaposiennes, Chang et al. [2] ont tout récemment mis en évidence par amplification différentielle à partir d'ADN extrait de tissu kaposien provenant d'un patient infecté par le VIH, deux séquences nucléotidiques, KS 330 et KS 631. Le clonage et le séquençage de ces séquences a permis de conclure à de fortes homologies $(50 \%)$ avec l'ADN codant pour certaines protéines des virus Epstein Barr et Saimiri. La mise en évidence de ces séquences a été effectuée par PCR dans 90 à $100 \%$ de lésions de SK, quel qu'en soit le type, résultats confirmés par l'ensemble de la communauté scientifique $\left(\mathrm{m} / \mathrm{s} n^{\circ} 6\right.$, vol. 11, p. 914; $n^{\circ} 10$, vol. 11, p. 16(05) $[33,34]$ et illustrés sur la figure 2. La détection par PCR de séquences de ce nouveau virus appelé Kaposi Sarcoma Herpes virus (KSHV) ou Human Iterpes Virus 8 ( HHV-8) dans les cellules mononucléées circulantes serait, chez les patients infectés par le VIH, corrélée au degré de l'immunosuppression et au risque de développer un SK [35]. Cela laisse penser que ce nouveau virus, HHV8 ou KSHV joue un rôle majeur, sinon exclusif, dans la physiopathologie du SK. Des résultats préliminaires de PCR in situ suggèrent que les séquences virales sont présentes, au sein des lésions, dans les cellules endothéliales et certaines cellules fusiformes dont le phénotype n'a pu encore être précisé [36]. Il semble, cependant, que les séquences virales soient inconstamment retrouvées par PCR dans les cellules fusiformes en culture au cours des premiers passages et en tout cas rapidement perdues durant les passages ultérieurs [37]. Il est possible que les conditions de culture des cellules kaposiennes ne permettent pas la survie des cellules infectées, ou encore que le virus soit cytolytique vis-à-vis du réservoir. Cela peut être rapproché des données établies avec des lignées de lymphomes. En effet, on a récemment établi des lignées cellulaires dérivées de lymphomes associés aux séreuses, co-infectées par EBV et HHV-8 [38], ou seulement par HHV8 [39]. Les résultats suggèrent la perte à long terme de la forme réplicative du virus dans une des lignées (BC-1) [40]. De plus, si la transmission du virus à partir de la lignée BC-1 à différents types cellulaires (lymphocytes, cellules épithéliales) est possible, celui-ci est ensuite perdu 


\section{RÉFÉRENCES}

36. Boshoff C, Schulz T, Kennedy M, Graham A, Fisher C, Thomas A, McGee J, Weiss R, O'Leary I. Kaposi's sarcoma-associated herpesvirus infects endothelial and spindle cells. Nature Med 1995; 1: 1274-8.

37. Lebbé C, De Crémoux P, Rybojad M Costa da Cunha C, Morel P, Calvo F. Kaposi's sarcoma and new herpesvirus. Lancet 1995; 345 : 1180 .

38. Cesarman E, Moore P, Rao P, Inghimari G, Knowles D, Chang Y. In vitro establishment and characterization of two acquired immunodeficiency syndrome-related lymphoma cell lines $\left(\mathrm{BC}_{-1}\right.$ and $\left.\mathrm{BC}-2\right)$ containing Kaposi's sarcoma-associated Herpesvirus-like (KSHV) DNA sequences. Blood $1995 ; 86: 2708-12$.

39. Renne R, Zhong W, Herndier B, McGrath M, Abbey N, Kedes N, Ganem D. Lytic growth of Kaposi's sarcoma-associated herpesvirus (human herpesvirus 8) in culture. Nature Med 1996; 2:341-6.

40. Moore P, Gao S, Dominguez G, Cesarman E, Lungu O, Knowles D, Garber R, Pelett P, McGeoch D, Chang Y. Primary characterization of a herpesvirus agent associated with Kaposi's sarcoma. J Virol 1996; $70: 549-58$.

41. Lunardi-Iskandar Y, Bryant J, Zeman R, Lam V, Samaniego F, Besnier J, Hermans P, Thierry A, Gill P, Gallo R. Tumorigenesis and metastasis of neoplastic Kaposi's sarcoma cell line in immunodeficient mice blocked by a human pregnancy hormone. Nature $1995 ; 375: 648$.

42. Ziegler J, Katongole-Mbidde E, Wabinga $\mathrm{H}$, Dollbaum CM. Absence of sex-hormone receptors in Kaposi's sarcoma. Lancet 1995; $345: 925$.

43. Stoica G, Hoffman J, Yuen P. Maloney murine sarcoma virus 349 induces Kaposi's sarcoma like lesions in balb c mice. $A m$ J Pathol 1990; 136: 933-47.

44. O'Connell K, Landman G, Farmer E, Edidin M. Endothelial cells transformed by SV40 T antigen cause Kaposi's sarcoma-like tumors in nude mice. Am J Pathol 1991 ; 139: 743-9.

45. Wittek A, Mitchell D, Armstrong G, Albini A, Martin G, Seemon R, Levenbook I, Wierenga D, Ridge J, Dunlop R, Lundquist M, steis M, Longo M, Muller J, Quinnan G. Propagation and properties of Kaposi's sarcoma-derived cell lines obtained from patients with AIDS: similarity of cultured cells to smooth muscle cells. AIDS 1991 ; au fur et à mesure des passages [40]. Beaucoup d'inconnues demeurent sur ce virus récemment visualisé en microscopie électronique [39], en particulier sa ou ses portes d'entrée, ses cibles et sites de latence.

\section{SK et hormones sexuelles}

tumeurs multicentriques proches du SK, comportant plusieurs populations cellulaires: celles exprimant l'antigène T de SV40 de phénotype endothélial et celles originaires de l'hôte, de phénotype fibroblastique [44]. Enfin Vogel et al. [25] obtiennent, après transfection du gène tat (sous le contrôle du VIH LTR), des souris trangéniques exprimant le gène tat au niveau de la peau, à l'exclusion de tout autre organe; $15 \%$ des mâles développent des tumeurs cutanées dont l'histologie est celle d'un SK mais n'exprimant pas le gène tat. A ce jour ce modèle n'a toujours pas été confirmé.

\section{Modèles cellulaires}

croissance des cellules kaposiennes in vitro mais également la formation in vivo, chez la souris beige nude, de tumeurs à partir de la lignée KSY-1. Ces résultats sont corrélés à la présence de récepteurs liant la $\beta$ HCG sur les cellules kaposiennes, in vitro et in vivo. On peut en rapprocher la rémission ou la stabilisation du SK durant la grossesse de deux patientes souffrant de SK associé au VIH, rapportées par les mêmes auteurs. L'ensemble de ces données demande confirmation; une étude récente ne met pas en évidence au sein des tissus kaposiens de récepteurs ni des androgènes, ni des oestrogènes, ni de la progestérone [42].

Les incertitudes sur l'origine de la cellule kaposienne, et tout particulièrement sur son caractère clonal, l'espoir de débouchés thérapeutiques ont conduit certaines équipes à tenter d'établir des modèles expérimentaux de SK.

\section{Modèles expérimentaux}

\section{Modèles animaux}

Certains modèles développés chez la souris ou chez le rat se rapprochent par leur caractère multifocal et leur histologie du SK.

Des souris BalbC, infectées par un rétrovirus ayant intégré l'oncogène murin mos, développent des tumeurs multifocales proches histologiquement du SK et exprimant la lectine ulex europeus agglutinin (UEA) [44]. Chez la souris nude, l'injection d'une lignée de cellules endothéliales murines infectées par le virus simien SV40 entraîne l'apparition de
Les caractéristiques des cultures cellulaires développpées à partir de tissus kaposiens ([1, 45-49] et Lebbé C, de Crémoux P, Millot G, Podgorniak M, Verola O, Calvo F, communication personnelle) sont détaillées dans le Tableau I. On peut retenir qu'à l'exception de deux lignées, les cellules kaposiennes ne sont pas des cellules transformées. Leur caractérisation immunohistochimique ou histochimique conduit à des résultats variables selon les équipes, illustrés sur les figures $1 C$ et $1 D$. Les lignées établies par certains [1] présentent, dans une proportion variable de cellules, certains marqueurs des cellules endothéliales (EN-4, UEA, récepteur des LDL acétylées, enzyme de conversion de l'angiotensine) mais ne synthétisent pas les antigènes de classe II du complexe majeur d'histocompatibilité ni le facteur Willebrand ou l'antigène PAL-E; elles produisent l' $\alpha$ actine de muscle lisse, caractéristique de la cellule musculaire lisse ou des myofibroblastes. Huang et al. (1993) [46] montrent, en revanche, que $10 \%$ à $50 \%$ des cellules, in vitro, expriment un phénotype de cellule dendritique dermique. Nos résultats suggèrent également une hétérogénéité cellulaire puisque $10 \%$ à $20 \%$ des cellules produisent au cours des premiers passages $(n<4)$ des marqueurs endothéliaux, $30 \%$ à $40 \%$ produisent durablement des marqueurs musculaires lisses, les autres cellules ayant un phénotype fibroblastique.

Ces cellules kaposiennes en culture synthétisent certains facteurs de 
CELLULES DÉRIVÉES DE SARCOME DE KAPOSI $\left(\mathrm{VIH}^{+}\right.$ET VIH-) : CARACTÈRES PHÉNOTYPIQUES DES CELLULES EN CULTURE (WP = CORPS DE WEIBEL-PALADE)

\begin{tabular}{|c|c|c|c|c|c|c|c|c|}
\hline & Delli Bovi* & Nakamura* & Roth* & $\begin{array}{l}\text { Herndier } \\
\text { [47] }\end{array}$ & Corbeil* & $\begin{array}{l}\text { Wittek } \\
\text { [45] }\end{array}$ & $\begin{array}{l}\text { Lunardi } \\
\text { [49] }\end{array}$ & Lebbé** \\
\hline Morphologie & fusiforme & fusiforme & fusiforme & $\begin{aligned} & \text { fusiforme } \\
+ & \text { épithélioïde }\end{aligned}$ & $\begin{aligned} & \text { fusiforme } \\
+ & \text { épithélioïde }\end{aligned}$ & fusiforme & $\begin{aligned} & \text { fusiforme } \\
+ & \text { épithélioïde }\end{aligned}$ & fusiforme \\
\hline $\begin{array}{l}\text { Ultrastructure } \\
\text { (corps de WP) }\end{array}$ & ND & - & ND & + & ND & - & ND & ND \\
\hline Caryotype & anormal & normal & normal & normal & ND & ND & anormal & normal \\
\hline Virus & - & - & - & $\mathrm{CMV}$ & - & (rétrovirus) & - & HHV8+/- \\
\hline $\begin{array}{c}\text { Pouvoir } \\
\text { angiogénique }\end{array}$ & ND & + & + & ND & ND & - & + & ND \\
\hline $\begin{array}{l}\text { Tumorigénicité } \\
\text { (souris nude) }\end{array}$ & ND & - & - & + & ND & - & + & - \\
\hline $\begin{array}{c}\text { Temps } \\
\text { de doublement }\end{array}$ & $24-30 \mathrm{~h}$ & $30-43 \mathrm{~h}$ & $52 \mathrm{~h}$ & ND & $24-36 \mathrm{~h}$ & ND & ND & $48 \mathrm{~h}$ \\
\hline Longévité & $70 p$ & $>60 p$ & $<52 p$ & $>50 p$ & ND & ND & $>100 p$ & ND \\
\hline $\begin{array}{l}\text { Croissance } \\
\text { en agar }\end{array}$ & $\begin{array}{c}\text { doublements } \\
\text { ND }\end{array}$ & ND & - & + & ND & + & + & - \\
\hline
\end{tabular}

Les cellules sont obtenues, soit après digestion enzymatique de tissu kaposien (poumon ou peau) soit directement à partir de liquide d'épanchement pleural ou d'ascite ou, plus rarement, par la technique des explants [1]. Les cultures sont effectuées sur matrices de fibronectine ou de gélatine mais parfois directement sur plastique, en présence de sérum de veau fotal, de FGF acide et d'héparine le plus souvent, ou de surnageant de culture de lymphocytes infectés par HTLV-II [1]; ces techniques permettent d'obtenir des cellules ayant le plus souvent une morphologie fusiforme, plus rarement épithélioïde, formant des monocouches adhérentes. La malignité de ces cellules est faible comme en témoigne leur caryotype, normal lorsqu'il est précisé à l'exception des résultats de Delli Bovi et al. * et de la lignée KSY-1 [48]. Les cellules conservent la propriété d'inhibition de contact ; le temps de doublement, pas toujours précisé, est lent (24 à 52 heures) et s'accroît au fur et à mesure des passages. Le nombre de passages est limité (15-20 passages), à l'exception de la lignée KSY-1; grâce à l'addition au milieu de culture de surnageant de culture de lymphocytes infectés par des rétrovirus et notamment HTLV-II, Salahuddin et al. * obtiennent un nombre quasi illimité de passages (> 60 passages). Ces cellules ne peuvent croître en milieu semi-solide, sauf pour deux lignées qui ont également un pouvoir tumorigène chez la souris nude [47, 49] ND: non déterminé; p: passages en culture.

* Référence citée dans Roth et al. (1992) [1].

** Résultats non publiés. 
Tableau II

CELLULES DÉRIVÉES DE SK (HIV+ ET HIV-) : SYNTHĖSE ET SÉCRÉTION DE CYTOKINES OU FACTEURS DE CROISSANCE PAR LES CELLULES EN CULTURE

\begin{tabular}{|c|c|c|c|c|}
\hline $\begin{array}{l}\text { Cytokine ou facteur } \\
\text { de croissance }\end{array}$ & Ensoli* & Roth* & Corbeil* & Lebbé** \\
\hline $\mathrm{TNF} \alpha$ & - & ND & ++ & - \\
\hline TNF $\beta$ & - & ND & + & ND \\
\hline IL6 & +++ & ND & +++ & ++ \\
\hline $\operatorname{IL} 1 \alpha$ & + & ND & ND & $+1-$ \\
\hline IL $1 \beta$ & +++ & ND & +++ & ND \\
\hline TGF $\beta$ & ++ & ++ & ND & $+1-$ \\
\hline GM-CSF & + & ND & +++ & $+1-$ \\
\hline VEGF & ND & ND & ND & ++ \\
\hline Oncostatine & + & ND & ND & - \\
\hline PDGFA & + & ++ & + & ND \\
\hline PDGFB & + & - & + & ND \\
\hline M-CSF & - & ND & ND & ND \\
\hline Endothéline & ND & ND & ++ & ND \\
\hline $\mathrm{IL}-2$ & - & +++ & ND & - \\
\hline Interféron $\gamma$ & - & ND & + & - \\
\hline Interféron $\alpha$ & + & ND & ND & ND \\
\hline
\end{tabular}

* Référence citée dans Roth et al. (1992) [1].

** Résultats non publiés.

Bien que les résultats varient selon les équipes, reflétant des conditions expérimentales différentes, les cellules kaposiennes en culture synthétisent des cytokines et des facteurs de croissance dont certains, en particulier I'IL6 et le PDGF, jouent un rôle autocrine. (-) absence de synthèse détectable, $(+)$ synthèse faible inconstamment détectable, $(+)$ synthèse minimum détectable, (++) 5 à 10 fois la synthèse minimum détectable, $1+++)$ à 50 fois la synthèse minimum détectable. ND: non déterminé.

croissance (Tableau II) et notamment de grandes quantités d'interleukines 1 (surtout $\beta$ ) et 6 et, à moindre degré, du GM-CSF. Les résultats concernant TNF $\alpha$ et PDGF et TGF $\beta$ varient selon les équipes. Une étude par hybridation in situ, menée en parallèle sur les tissus kaposiens et les cellules en culture [50], a permis de mettre en évidence la synthèse de l'IL6 par les cellules en culture et dans les tissus, et une synthèse de l'IL $1 \beta$ et du PDGF-B par certaines sous-populations cellulaires seulement. Une forte production de VEGF par rapport à des cellules témoins (cellules musculaires lisses ou cellules endothéliales) a été rapportée [51]. Les résultats concernant la synthèse des FGF sont disparates. La synthèse de facteurs de croissance angiogènes pourrait expliquer l'effet angiogène des cellules cultivées sur membrane chorioallantoïde de poulet et l'apparition après injections de nude, de tumeurs transitoires d'origine murine richement vascularisées ([52] et communication personnelle). L'obtention de tumeurs vascularisées murines est également possible après injection, en présence de matrigel, de FGF basique et de protéine Tat, ou encore de surnageant de culture de cellules kaposiennes et de protéine Tat, ces résultats suggérant une synergie d'action entre Tat et le FGF basique [48, 52]. Les besoins en facteurs de croissance des cellules kaposiennes varient selon les équipes, ces différences étant probablement dues à l'hétérogénéité des conditions expérimentales. La nécessité du PDGF est controversée [1]. La croissance des cellules est, pour certains, stimulée par l'IL6 et la protéine Tat [1]. Les effets prolifératifs de l'oncostatine sur les cellules kaposiennes, suggérés par certains, n'ont pas été confirmés [50].

Les interactions entre cellules $\mathrm{T}$ infectées par des rétrovirus et cel- lules kaposiennes pourraient jouer un rôle très important dans la physiopathologie du SK via l'ILl $\alpha$ et $\beta$, les TNF $\alpha$ et $\beta$, le PDGF et, à un degré moindre, l'IL6 et le GM-CSF [53]. La protéine Tat agirait en synergie avec ces cytokines ou facteurs angiogéniques, tant sur les cellules kaposiennes que sur des cellules endothéliales normales in vitro. Les données actuelles suggèrent donc que le SK associé au SIDA, mais peut-être aussi les autres formes de la maladie, seraient liés à l'activation et à la prolifération de cellules mésenchymateuses (les futures cellules fusiformes) en réponse à différents facteurs de croissance et d'activation.

La mise au point de ces modèles a déjà permis de tester l'influence de différentes molécules (comme le SPPG, peptidoglycane polysaccharidique) ou cytokines (comme l'interféron $\alpha$ ) sur la croissance des cellules kaposiennes ou la formation de tumeurs chez la souris nude. Des 


\section{RÉFÉRENCES}

46. Huang Y, Friedman-Kien A, Li J, Nickoloff B. Cultured Kaposi's sarcoma cell lines express factor XIIIa, CD 14 and VCAM1, but not factor VIII or EIAM1. Arch Dermatol $1993 ; 129: 1291-6$.

47. Herndier B, Werner A, Arnstein P, Abbey N, Demartis F, Cohen R, Shuman M, Levy I. Characterization of a human Kaposi's sarcoma cell line that induces angiogenic tumors in animals. AIDS 1994; $8: 57,5-81$.

48. Albini A, Fontanini G, Masiello L, Tacchetti C, Bigini D, Luzzi P, Noonan D, Stetler-Stevenson $\mathrm{W}$. Angiogenic potential in vivo by Kaposi's sarcoma cell-free supernatants and HW-l tat product: inhibition of Kaposi's sarcoma-like lesions by tissue inhibitor of metalloproteinase-2. AIDS 1994; 8: $1237-44$

49. Lunardi-Iskandar $Y$, Gill P, Lam V, Zeman R, Michaels F, Mann D, Reitz M, Kaplan M, Berneman Z, Carter D, Bryant J, Gallo R. Isolation and characterization of an immortal neoplastic cell line (KS Y-1) from AIDS associated Kaposi's sarcoma. J Nall Cancer Inst 1995; 87 : 97481.

50. Stürzl M, Brandstetter H, Zietz C, Eisenberg B, Raivich G, Geaing D, Broch Meyer $N$, Hofschneider P. Identification of interleukin-l and PDGF-B as major mitogens for the spindle cells of Kaposi's sarcoma: a combined in vitro and in vivo analysis. Oncogene $1995 ; 10: 2007-16$.

51. Weindel K, Marmé D, Weich H. AIDSassociated Kaposi's sarcoma cells in culture express vascular endothelial growth factors. Biochem Biophys Res Commun 1992; 183 : $1167-74$.

52. Ensoli B, Gendelman R, Markham $P$, Fiorelli V, Colombini S, Raffeld M, Cafaro A, Chang H, Brady J, Gallo R. Synergy between fibroblast growth factor and HIV-1 tat protein in induction of Kaposi's sarcoma. Nature 1994; 371 : 674-80

53. Barillari G, Buonaguro L, Fiorelli V, Hoffman L, Michaels F, Gallo R, Ensoli B. Effects of cytokines from activated immune cells on vascular cell growth and HIV-1 gene expression. J Immunol 1992; 149 : 3727-34 molécules anti-angiogéniques (inhibiteurs des métalloprotéases, oligonucléotides antisens anti-PDGF, antiFGF ou anti-VEGF) pourraient également avoir des débouchés thérapeutiques intéressants.

En conclusion, les principales avancées dans la physiopathologie de la maladie de Kaposi concernent la mise en évidence, grâce aux modèles expérimentaux, du rôle de diverses cytokines et facteurs de croissance sécrétés de façon autocrine ou paracrine et, surtout, l'isolement d'un nouveau virus, $\mathrm{HHV}-8$ dans les tissus kaposiens. Les recherches visent actuellement à déterminer, au sein des lésions kaposiennes, les cellules réservoir de ce virus dont on ignore encore le mode de transmission et les sites de latence éventuels

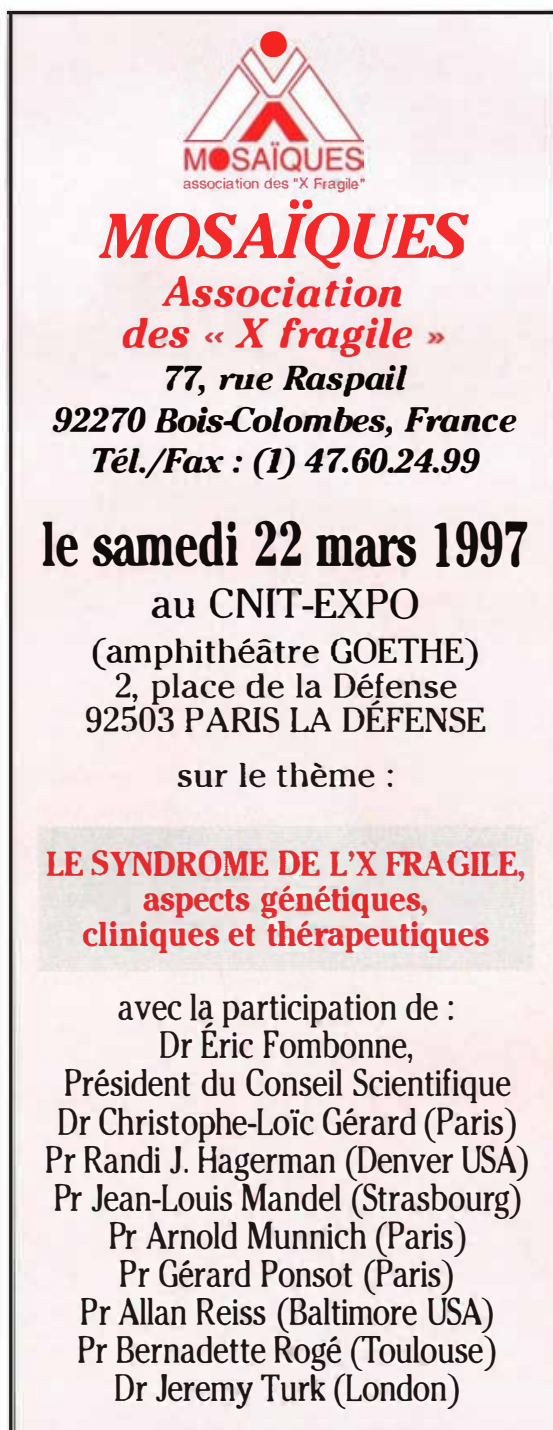

\section{Summary}

Cellular and molecular characteristics of Kaposi's sarcoma

Kaposi's sarcoma (KS) is a vascular neoplasia with four clinico-epidemiological settings. The histogenesis of the lesions, characterized by the prolif eration of spindle-shaped cells, is controversial : endothelial, dendritic or smooth muscle derived? The nature of KS, true clonal origin or hyperplasia, is still debated. Recent data suggest that KS is a cytokine induced benign proliferation which could occasionally transform itself into a true malignant tumor. Although strong evidences favor the role of an infectious agent in this disease, virological studies have failed to conclude until the recent identification of a new herpes virus, HHV8 , from KS lesions. The role of this new virus in the pathogenesis of $\mathrm{KS}$ is probable since blood detection of HHV-8 sequences is predictive of development of KS in HIVinf ected patients. No reproducible in vivo model of $\mathrm{KS}$ is available but in vitro models have been developed aiming at a better understanding of KS pathogenesis and of antiproliferative effects of various pharmacological agents.

\section{Accès à la base de donnée internationale en Immunogénétique : IMGT/LIGM-DB}

La base de données IMGT/LIGM-DB (Immunoglobulines et Récepteurs T) qui, avec HLA-DB (Julia Bodmer, ICFR, Londres), appartient à la base de données internationale ImMunoGeneTics IMGT, est accessible, depuis le 10 juillet 1995, sur le serveur WWW du CNUSC (http://imgt.cnusc.fr:8104). IMGT/LIGM-DB contient à ce jour plus de 9000 séquences $(6860$ séquences d'immunoglobulines et 2410 de récepteurs T) de 61 espèces différentes. Les fichiers à plat des séquences annotées (900) sont accessibles sur les serveurs ftp anonyme (ftp.ebi.ac.uk/ pub/databases/imgt) et WWW (ftp://ftp.ebi.ac. uk/pub/databases/imgt) d'EMBL-EBI, depuis le 24 juin 1995.

Contact :

Pr. Marie-Paule Lefranc, Coordinateur de IMGT Tél. : 67.61.36.34. Fax : 67.04.02.31/45.

E-mail : lefranc@ligm.crbm.cnrs-ınop.fr 